\title{
Fibrin - Alginat als Matrix im Tissue Engineering
}

Georg Matziolis,

Carsten Perka,

Sebastian Stern

Schlagworte:

Fibrin - Alginat -

Tissue engineering
In der Orthopädie werden zunehmend autologe Knorpeltransplantationen durchgeführt. Die Entnahmemorbidität könnte dabei durch die Herstellung synthetischer zelltragender Gewebeersatzmaterialien entscheidend gesenkt werden.

Keine der zur Zeit verfügbaren Materialien erfüllt sowohl klinische als auch biologische Anforderungen an eine "ideale" Zellmatrix.

Wir konnten durch Mischung zweier unterschiedlicher Substanzen, Fibrin und Alginat, unter Ausnutzung ihrer spezifischen Vorteile in vitro und in vivo, eine 3-dimensionale humane Chondroblastenkultur etablieren.

Im Gegensatz zu einer reinen Fibrinmatrix, blieb der Zelldifferenzierungsgrad über 30 Tage erhalten, so dass knorpelspezifische Matrixsubstanzen (Kollagen II, Proteoglykane) synthetisiert werden konnten. Nach Entfernung des Alginats waren $95 \%$ der Zellen vital.

Wir folgern, dass durch die Verwendung einer Fibrin-Alginat Mischmatrix kartilaginäre Konstrukte in vitro herstellbar sind. Das Alginat kann ohne Schädigung der Chondroblasten entfernt werden, so dass biokompatible und -resorbierbare Chondroblastenkonstrukte transplantiert werden können.

\section{Einleitung}

Die zunehmende Zahl autologer Knorpeltransplantationen in der Orthopädie führt zu einer relevanten Entnahmemorbidität, deren Senkung ein Hauptziel des Tissue Engineering ist. Dazu ist die Herstellung zelltragender transplantierbarer Konstrukte erforderlich, was eine dreidimensionale Zellkultivierung bedingt.

Die Eigenschaften einer "idealen" synthetischen Matrix ergeben sich sowohl aus biologischen als auch klinischen Erfordernissen (Tab. 1).

\begin{tabular}{|l|l|}
\hline Biologische Sicht & Klinische Sicht \\
\hline gute Adhäsion der Zellen & Biokompatibilität \\
\hline $\begin{array}{l}\text { Gleichmäßige Zellverteilung } \\
\text { im Raum }\end{array}$ & hohe Primärstabilität \\
\hline $\begin{array}{l}\text { Gewährleistung der } \\
\text { Zellnutrition einschließlich der } \\
\text { Diffusion von Abbauprodukten }\end{array}$ & gutes Handling \\
\hline Erhalt der Zelldifferenzierung & einfache operative Fixierung \\
\hline Stimulation der Zellproliferation & Bioresorbierbarkeit \\
\hline Stimulation der Matrixneosynthese & Bezahlbarkeit \\
\hline
\end{tabular}

Tab. I: In Anlehnung an die aktuelle Literatur zusammengefasste Eigenschaften einer "idealen" Matrix für das Tissue Engineering
Das Fehlen eines standardisierten dreidimensionalen Zellkultursystems spiegelt wieder, dass die zur Zeit verfügbaren Matrix - Substanzen lediglich Kompromisse mit jeweils spezifischen Vorteilen in vitro oder in vivo darstellen (Tab. 2).

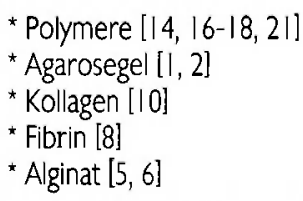

Tab. 2: In vitro und in vivo etablierte Matrix Substanzen

Wir konzentrieren uns in der vorliegenden Arbeit auf die etablierten Substanzen Fibrin und Alginat, da sie aufgrund ihrer konträren Eigenschaften in vivo und in vitro die Möglichkeit eines Synergismus bieten.

Während Fibrin als körpereigenes Produkt der Blutgerinnung biokompatibel und -resorbierbar ist, besitzt zur Zeit verfügbares Alginat antigene Eigenschaften die regelhaft $z u$ Entzündungsreaktionen führen $[3,4,22]$. In vitro hingegen ist eine Alginatmatrix Fibrin überlegen, da sie den Differenzierungsgrad der Zellen erhalten kann [6], wohingegen in eine Fibrinmatrix eingebettete Zellen zu fibrozytärer Dedifferenzierung neigen [8]. 
Ziel der vorliegenden Arbeit war die Herstellung und Charakterisierung einer Fibrin-Alginat Mischmatrix, um die Vorteile von Alginat in vitro zu Nutzen es jedoch vor einem Einsatz in vivo aus den Mischkonstrukten ohne Schädigung der Zellen zu entfernen. Dazu wurden ChondroblastenFibrin-Alginat Mischkonstrukte ebensolchen nach Entfernung des Alginats gegenübergestellt und hinsichtlich Zellproliferation, -vitalität, -differenzierung und knorpelspezifischer Matrixsynthese untersucht.

\section{Material and Methoden \\ Zellextraktion}

Chondroblasten wurden aus Knorpelgewebe, welches bei knieendoprothetischen Operationen anfiel, extrahiert. Das Gewebe wurde nach mechanischer Zerkleinerung in eine 0,4 \%ige Pronaselösung gegeben. Nach 60 Minuten wurden die Gewebsfragmente in eine Mischung aus 0,05\% Kollagenase, $5 \%$ fetalem Kälberserum (FCS), $100 \mathrm{IU} / \mathrm{ml}$ Penicillin, $100 \mu \mathrm{g} / \mathrm{ml}$ Streptomycin und $5 \mu \mathrm{g} / \mathrm{ml}$ Amphotericin in Ham's F12 Nährmedium überführt. 18 Stunden später wurde das Zelllysat filtriert ( $52 \mu \mathrm{m}$ - Polyesterfilter), zentrifugiert und mit PBS gewaschen.

\section{Herstellung der Fibrin - Alginat Mischmatrix}

Fibrinogenlösung (TISSUCOL - Duo $S^{\oplus}$ Immuno, Baxter Deutschland) und Alginat (Sigma, USA) wurden zu Endkonzentrationen von $4,5 \%$ respektive $0,6 \%$ vermengt und darin die gewaschenen Zellpellets (je ca. 600.000 Zellen) mit einem Endvolumen von $800 \mu \mathrm{l}$ Volumen aufgelöst. Das Chondroblasten-Fibrinogen-Alginat Gemisch polymerisierte in $100 \mathrm{mmol} / \mathrm{l} \mathrm{Kalziumchloridlösung} \mathrm{mit} 46 \mathrm{IU} / \mathrm{ml}$ Thrombin (TISSUCOL - Duo $\mathrm{S}^{\circledR}$ Immuno, Baxter, Deutschland) zu formstabilen Konstrukten. Diese wurden zunächst in 0,15 $\mathrm{mmol} / \mathrm{l}$ Kochsalzlösung dann in Ham's F12 Medium gewaschen (Gruppe 1).

Nachfolgend wurde das Alginat aus den Konstrukten durch Entfernung der Kalziumionen durch Chelatbildner (55 $\mathrm{mmol} / \mathrm{l}$ Natriumzitrat, $150 \mathrm{mmol} / 1$ Natriumchlorid, 30 $\mathrm{mmol} / \mathrm{l}$ EDTA) gelöst (Abb. 1), so dass ChondroblastenFibrin Konstrukte resultierten (Gruppe 2).

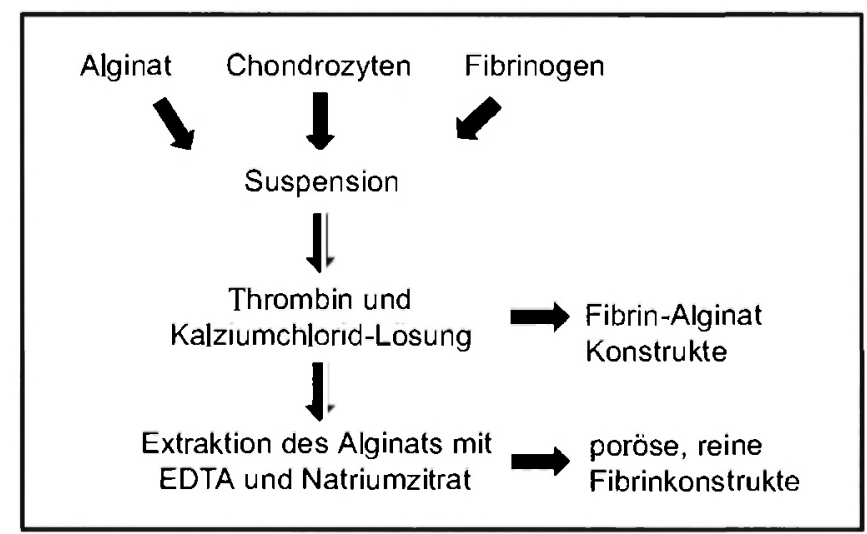

Abb. I: Schematische Darstellung der Herstellung einer Fibrin-Alginat Mischmatrix

\section{Zellkultur}

Die Konstrukte wurden in Ham's F12 Medium, $10 \%$ FCS, $100 \mathrm{IU} / \mathrm{ml}$ Penicillin, $100 \mu \mathrm{g} / \mathrm{ml}$ Streptomycin und $5 \mu \mathrm{g} / \mathrm{ml}$ Amphotericin bei $37{ }^{\circ} \mathrm{C}$ und $3,5 \% \mathrm{CO}_{2}$ kultiviert. Zur Stabilisierung der Fibrinkonstrukte wurden dem Nährmedium 12 $\mathrm{kIU} / \mathrm{ml}$ Aprotinin (Trasylol ${ }^{\circledR}$, Bayer, Deutschland) hinzugegeben.

\section{Histologie}

Die Konstrukte wurden nach 5, 20 und 30 Tagen mit flüssigem Stickstoff schockgefroren und bis zur Auswertung bei $-140{ }^{\circ} \mathrm{C}$ gelagert. Zur histologischen Aufarbeitung wurden $5 \mu \mathrm{m}$ dicke Schnitte mit einem Kryostaten angefertigt, mit Hämatoxylin/Eosin (HE) oder Alcian Blau gefärbt und lichtmikroskopisch ausgewertet. Morphologisch erfolgte sowohl anhand der HE - Schnitte als auch unter dem Phasenkontrastmikroskop eine quantitative Determinierung zwischen chondroblastären und fibrozytären Zellformen.

\section{Immunhistologie}

Die Schnitte wurden immunhistologisch auf Kollagen I, II und Proteoglykanen hin untersucht. Dazu wurden sie mit 3-Aminotriethoxysilan (Sigma, USA) beschichtet [7]. Nach Trocknung der Präparate wurden die Präparate mit Acetaldehyd ( 5 Minuten, $20^{\circ} \mathrm{C}$ ) fixiert und mit den jeweiligen Antikörpern (Chemicon, USA) inkubiert. Die Immunfärbung erfolgte mittels biotinyliertem Kaninchen-Anti-Maus Antikörper, alkalische Phosphatase-konjugiertem Streptavidin und dem Neofuchsin Substrat System (Dako, Dänemark).

Transmissionselektronenmikroskopie

Zur Transmissionselektronenmikroskopie (TEM) wurden die Konstrukte mit 2,5\% Glutaraldehyd gefolgt von $1 \% \mathrm{OsO}_{4}$ und 1,5\% Ferrozyanid fixiert und in Epon Resin eingelegt. Die Schnitte wurden mit einem Ultramikrotom angefertigt und mit Uranylacetat kontrastiert.

\section{Statistische Analyse}

$\mathrm{Zu}$ den quantitativ erhobenen Daten wurden arithmetischer Mittelwert sowie Standardabweichung ermittelt. Die Signifikanzanalyse erfolgte mit dem Mann-Whitney-U Test wobei das Signifikanzniveau auf $p<0,05$ festgelegt wurde.

\section{Ergebnisse}

Sämtliche Konstrukte waren über den Beobachtungszeitraum von 30 Tagen im Medium formstabil. Überdies kam es zu keinem signifikanten Masseverlust.

Innerhalb von 20 Tagen stieg die Zelldichte von initial 7,6 x $10^{5} / \mathrm{ml}$ auf $3,6 \times 10^{6} / \mathrm{ml}$ (Gruppe 1) respektive $5,1 \times 10^{6} / \mathrm{ml}$ (Gruppe 2) an (Abb. 2). Die Anzahl vitaler Zellen lag dabei stets über $95 \%$.

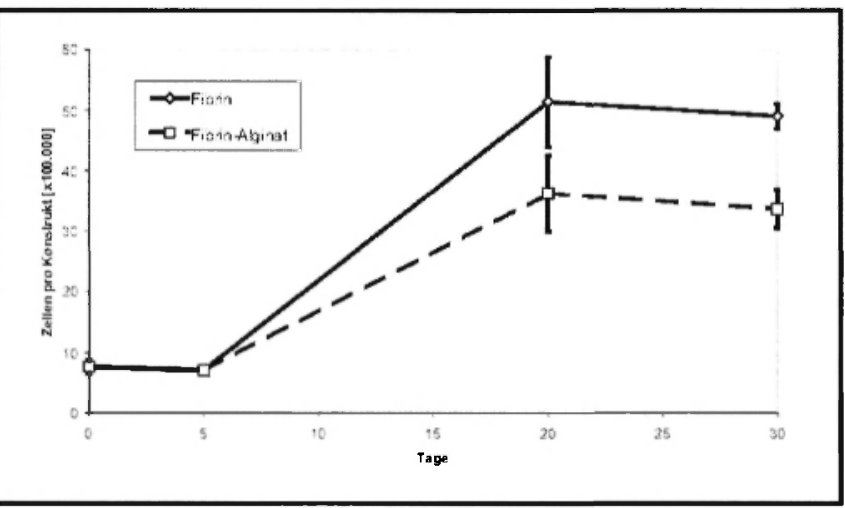

Abb. 2: Zellproliferation der Fibrin-Alginat und der reinen Fibrin Konstrukte. Die Zellvitalität lag stets über $95 \%$.

\section{Fibrin Konstrukte}

Bei der histologischen Untersuchung wiesen in der reinen Fibringruppe $20 \%$ der Zellen schon nach fünf Tagen morphologische Kriterien der Dedifferenzierung zu fibrozytären 
Formen auf (Verlust der Kugelform und pseudopodienartige Zytoplasmaaussackungen). Mit zunehmender Zeit stieg der relative Anteil dieser Zellform an der Gesamtzellzahl mit einem Maximum nach 30 Tagen, was auf eine höhere Proliferationsrate im Vergleich zu differenzierten Chondroblasten zurückzuführen war. Dies korrelierte mit einer geringeren Matrixsyntheseleistung der dedifferenzierten Zellen, welche sich in der negativen Alcian Blau Färbung ihrer Interzellularsubstanz wiederspiegelte. Die immunhistologische Untersuchungen wiesen einen geringen Anteil an Proteoglykanen bei vollständigem Fehlen von Kollagen I und II nach.

Chondroblasten bildeten wenige große Zellhaufen und ließen sich innerhalb des Konstrukts sowohl zentral als auch peripher nachweisen. Die Matrix war lediglich innerhalb dieser Cluster Alcian Blau positiv.

\section{Fibrin-Alginat Konstrukte}

Im Gegensatz dazu behielten in der Fibrin-Alginat Gruppe mehr als $90 \%$ der Zellen ihre chondroblastäre Differenzierung. Ihr Anteil nahm mit der Zeit aufgrund einer abnehmenden Zahl dedifferenzierter Zellen zu, welche sich nach 30 Tagen größtenteils fragmentiert und avital zeigten. In der Alcian Blau Färbung zeigte sich eine höhere knorpelspezifische Matrixsynthese der gesamten Konstrukte im Vergleich zur reinen Fibringruppe. Immunhistologisch konnte eine hohe Konzentration der knorpelspezifischen Matrixbestandteile Proteoglykan und Kollagen II nachgewiesen werden [Abb. 3]. Sämtliche Konstrukte zeigten sich in der Kollagen I Färbung negativ.

Die Chondroblasten konzentrierten sich im Zentrum der Konstrukte zu Zellclustern, welche in der TEM interzelluläre Kollagenfibrillen aufwiesen.

Die Koexistenz unterschiedlicher Differenzierungsformen der Zellen innerhalb der Konstrukte konnte für beide Gruppen zu den verschiedenen Beobachtungszeitpunkten in Übereinstimmung mit den lichtmikroskopischen Befunden durch die TEM bestätigt werden.

\section{Diskussion}

Im Tissue Engineering besteht die Notwendigkeit der dreidimensionalen Zellkultivierung, welche zur Einbettung der Zellen eine synthetische Matrix erfordert. Aufgrund der nur einseitigen Adhäsionsmöglichkeit, bilden Zellen in einem Monolayer pseudopodienartige Zytoplasmaaussackungen. Dies führt neben der morphologischen, über die Zeit auch zu einer funktionellen Dedifferenzierung der Zellen mit einem Verlust spezifischer Eigenschaften und Syntheseleistungen. Während jedoch die Techniken der zweidimensionalen Zellkultur weitgehend standardisiert sind, stellt der Übergang zu Zell-Matrix Konstrukten noch eine Herausforderung dar, da bislang keine Trägersubstanz verfügbar ist, die allen Forderungen an eine "ideale" Matrix gerecht wird.

Ziel ist neben einer Zunahme der Zellzahl vor allem die Synthese einer gewebsspezifischen Matrix, was einen Erhalt des Differenzierungsgrades der Zellen über mehrere Generationen voraussetzt.

Wesentliche Anforderungen der Klinik sind Bezahlbarkeit, Verfügbarkeit, einfaches Handling, mechanische Stabilität, Biokompatibilität und Resorbierbarkeit.

Wir haben versucht durch Kombination der etablierten Matrixsubstanzen Fibrin und Alginat eine Mischmatrix mit überlegenen Eigenschaften zu synthetisieren.

Bei Fibrin handelt es sich um eine klinisch etablierte, biokompatible und resorbierbare Substanz. Diesen vorteilhaften Eigenschaften steht aus biologischer Sicht die Tatsache gegenüber, dass Chondrozyten in Fibrin zu fibrozytären
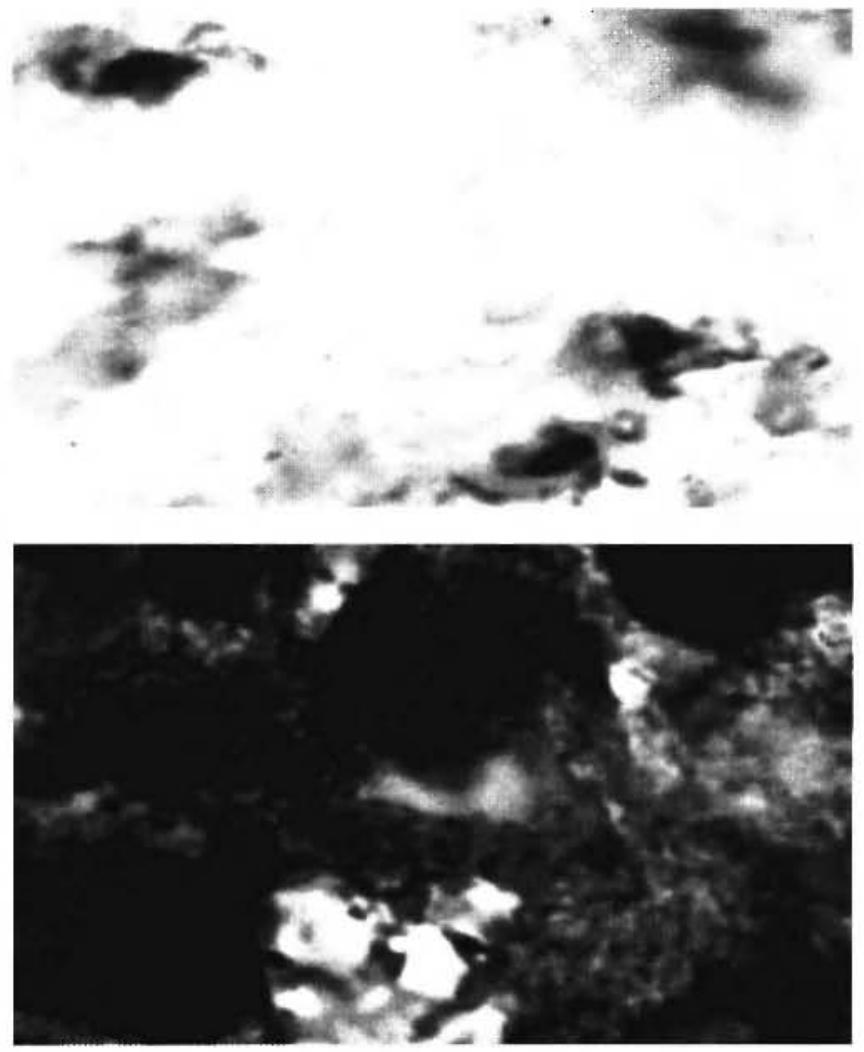

Abb. 3a, b: Immunhistologischer Nachweis von Proteoglykanen (a) und Kollagen II (b) in Fibrin-Alginat Mischkonstrukten [x 40]

Formen dedifferenzieren [8], so dass sie in Übereinstimmung mit unseren histologischen und immunhistologischen Ergebnissen die Fähigkeit verlieren eine eigene Knorpelmatrix zu synthetisieren.

Alginat geliert als lineares Polysaccharid unter dem Einfluss divalenter Kationen wie Kalzium oder Barium [15]. Es besitzt im Vergleich zum Fibrin konträre Eigenschaften. In klinischen Versuchen konnten die unproblematische Handhabung sowie eine hinreichende mechanische Stabilität nachgewiesen werden [12]. Dabei vermag es den Differenzierungsgrad von eingebetteten Chondrozyten in vitro über lange Zeit zu erhalten [6]. Die wesentlichen Nachteile von Alginat im klinischen Einsatz liegen in der mangelnden Biokompatibilität und Resorbierbarkeit, was in vivo zu Entzündungsreaktionen mit konsekutiven Fibrosen führt [3, 22]. Es wird diskutiert, dass Verunreinigungen für die relative biologische Unverträglichkeit verantwortlich sind [4], so dass Bestrebungen bestehen, den Reinheitsgrad von Alginat durch Optimierung des Produktionsprozesses zu steigern [9, 11]. In der vorliegenden Arbeit wurde ein anderer Lösungsansatz verfolgt.

Dabei wurde die differenzierungserhaltende Eigenschaft des Alginats während der Zellproliferation und Synthese einer spezifischen Zellmatrix ausgenutzt. Dies geschah im Rahmen von Mischkonstrukten aus Fibrin und Alginat. Unsere Ergebnisse zeigen, dass es dabei zur Synthese einer knorpelspezifischen Matrix, nachgewiesen anhand von Kollagen II, Proteoglykanen und einer positiven Alcian Blau Reaktion, kam.

Wir konnten nachweisen, dass durch den Einsatz einer Mischmatrix aus Fibrin und Alginat Konstrukte synthetisierbar sind, welche die Differenzierung von Chondrozyten zu erhalten vermögen. In vorhergehenden Arbeiten konnten 
ähnlich positive Eigenschaften für Periostzellen [19] und Nucleus pulposus-Zellen [20] aufgezeigt werden.

Nach Erreichen einer hinreichenden Zelldichte und Matrix kann, vor einem Einsatz in vivo, das Alginat ohne Schädigung der Zellen herausgelöst werden, so dass man biokompatible und resorbierbare poröse Fibrinkonstrukte erhält.

Wir sehen den Hauptvorteil unseres Vorgehens in der Übertragung von in eigensynthetisierter Matrix eingebetteten, differenzierten Zellen. Dies kann weder durch den Einsatz einer reinen Fibrin- noch einer Alginatmatrix realisiert werden.

Die Überlegenheit dieses Prinzips gegenüber konventionellen Trägersystemen konnte sowohl für Knorpel- [13] als auch Knochendefekte [13] in vivo gezeigt werden.

Solange keine neuen Biomaterialien verfügbar sind, ist die geschickte Kombination etablierter Matrixsubstanzen mit ihren bekannten Vor- und Nachteilen eine evolutionäre Möglichkeit, sich dem "idealen" Trägersystem des Tissue Engineering zu nähern.

\section{Abstract}

Autologous tissue transplantation has become a standard procedure in orthopaedic surgery. Tissue engineering may help minimizing donor side morbidity by transplantation of synthetic cell embedded constructs.

While methods of 2-dimensional cell culture are standardized, techniques of 3-dimensional cell culture are mostly experimental. All established matrix substances have specific advantages in vitro or in vivo, none fulfilling all conditions of an "ideal" synthetic cell matrix. We focussed on the well established matrix substances fibrin and alginate.

Fibrin is a product of haemostasis and fully biocompatible and resorbable, that are great advantages for an use in vivo. In contrast it conducts a dedifferentiation of embedded cells to fibrocyte-like phenotypes in vitro, so it may only be used as a carrier for transplantation but not as a matrix for cell proliferation.

Alginate is a linear polysaccharide, forming a gel when getting in contact to calcium ions. It can serve as a synthetic cell matrix for 3-dimensional cultivation in vitro, as embedded cells don't dedifferentiate and synthesise tissue specific matrix. A great disadvantage of alginate is its bioincompatibility, at least in up to now available purity, leading to inflammation and consecutive fibrosis in vivo.

We combined the advantages of alginate in vitro with those of fibrin in vivo by using a fibrin-alginate mixed matrix. It was synthesised by adding alginate to fibrinogen - both in aqueous solution, then dissolving a cell pellet and letting this construct polymerize in a calcium and thrombin solution. Afterwards the alginate component could be washed out of the constructs in a sodium citrate and EDTA solution.

We compared the fibrin-alginate constructs with porous fibrin constructs synthesized out of fibrin-alginate constructs by removing the alginate component. Cell proliferation, vitality, differentiation and cartilage specific matrix synthesis were quantified using cell counting and characterisation by transmission electron microscopy (TEM), histological and immunohistochemical $(\mathrm{IH})$ methods.

Our results show, that a fibrin-alginate matrix preserves the cell differentiation of chondroblasts over an observing period of 30 days, allowing a 4.7 fold increase of cell number at a viability index of $95 \%$. IH revealed the synthesis of a cartilage specific matrix positive for collagen II and proteoglycans. TEM confirmed these results showing chondroblast specific cell phenotypes and the extracellular presence of collagen fibrils.
In contrast a fibrin matrix lead to a dedifferentiation of the embedded chondroblasts to fibrocyte phenotype forms, having higher proliferation rates than the chondroblasts, leading to a decrease of cartilage specific cell ratio with time. These morphological results corresponded with the $\mathrm{IH}$ and TEM findings that showed only few clusters of cartilage specific matrix synthesising chondroblasts.

We conclude that chondroblasts can be cultured in a fibrinalginate mixed matrix keeping their differentiation and allowing them to synthesise a cartilage specific matrix. The alginate component can be removed without compromising the embedded cells before transplantation, leading to biocompatible and resorbable constructs.

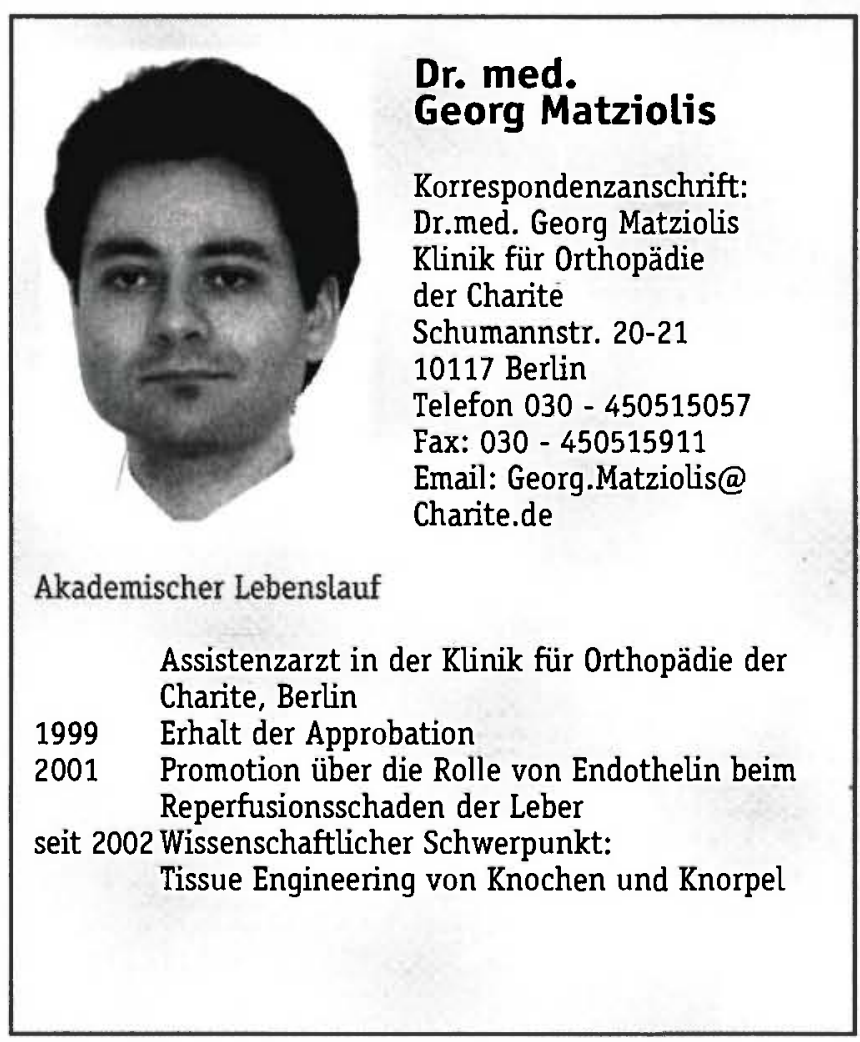

\section{Literatur}

[1] Balgude A. P., Yu X., Szymanski A., Bellamkonda R.: (2001) Agarose gel stiffness determines rate of DRG neurite extension in 3D cultures. Biomaterials 22:1077-1084

[2] Bellamkonda R., Ranieri J. P., Bouche N., Aebischer P.: (1995) Hydrogel-based three-dimensional matrix for neural cells. J.Biomed.Mater.Res. 29:663-671

[3] Bhalla R. K., Murphy J., Jones T. M., Roland N. J.: (2002) Foreign body reaction to calcium alginate fibre mimicking recurrent tumour of the submandibular salivary gland. Br.J.Oral Maxillofac.Surg. 40:172-174

[4] De Vos P., De Haan B. J., Wolters G. H., Strubbe J. H., Van Schilfgaarde R.: (1997) Improved biocompatibility but limited graft survival after purification of alginate for microencapsulation of pancreatic islets. Diabetologia 40:262-270 
[5] Hauselmann H. J., Aydelotte M. B., Schumacher B. L., Kuettner K. E., Gitelis S. H., Thonar E. J.: (1992) Synthesis and turnover of proteoglycans by human and bovine adult articular chondrocytes cultured in alginate beads. Matrix $12: 116-129$

[6] Hauselmann H. J., Fernandes R. J., Mok S. S., Schmid T. M., Block J. A., Aydelotte M. B., Kuettner K. E., Thonar E. J.: (1994) Phenotypic stability of bovine articular chondrocytes after long-term culture in alginate beads. J.Cell Sci. 107 ( Pt 1): $17-27$

[7] Hoffstrom B. G., Wayner E. A.: (1994) Immunohistochemical techniques to study the extracellular matrix and its receptors. Methods Enzymol. 245:316-346

[8] Homminga G. N., Buma P., Koot H. W., van der Kraan P. M., van den Berg W. B.: (1993) Chondrocyte behavior in fibrin glue in vitro. Acta Orthop.Scand. 64:441-445

[9] Jork A., Thurmer F., Cramer H., Zimmermann G., Gessner P., Hamel K., Hofmann G., Kuttler B., Hahn H. J., JosimovicAlasevic 0., Fritsch K. G., Zimmermann U.: (2000) Biocompatible alginate from freshly collected Laminaria pallida for implantation. Appl.Microbiol.Biotechnol. 53:224-229

[10] Kimura T., Yasui N., Ohsawa S., Ono K.: (1985) Biosynthesis of type IX collagen during chick limb development. Biochem.Biophys.Res.Commun. 130:746-752

[11] Klock G., Frank H., Houben R., Zekorn T., Horcher A., Siebers U., Wohrle M., Federlin K., Zimmermann U.: (1994) Production of purified alginates suitable for use in immunoisolated transplantation. Appl.Microbiol.Biotechnol. 40:638-643

[12] Mullen Y., Maruyama M., Smith C.V.: (2000) Current progress and perspectives in immunoisolated islet transplantation. J.Hepatobiliary.Pancreat.Surg. 7:347-357

[13] Perka C., Schultz 0., Spitzer R.S., Lindenhayn K., Burmester G.R., Sittinger M.: (2000) Segmental bone repair by tissue-engineered periosteal cell transplants with bioresorbable fleece and fibrin scaffolds in rabbits. Biomaterials 21:1145-1153

[14] Puelacher W. C., Mooney D., Langer R., Upton J., Vacanti J. P., Vacanti C. A.: (1994) Design of nasoseptal cartilage replacements synthesized from biodegradable polymers and chondrocytes. Biomaterials 15:774-778

[15] Seely G. R., Hart R. L. . (1974) The binding of alkaline earth metal ions to alginate. Macromolecules 7:706-710

[16] Sittinger M.: (1995) [Tissue engineering: artificial tissue replacement containing vital components]. Laryngorhinootologie 74:695-699

[17] Sittinger M., Bujia J., Minuth W. W., Hammer C., Burmester G. R.: (1994) Engineering of cartilage tissue using bioresorbable polymer carriers in perfusion culture. Biomaterials 15:451-456

[18] Sittinger M., Bujia J., Rotter N., Reitzel D., Minuth W. W., Burmester G. R.: (1996) Tissue engineering and autologous transplant formation: practical approaches with re- sorbable biomaterials and new cell culture techniques. Biomaterials 17:237-242

[19] Spitzer R. S., Perka C., Lindenhayn K., Zippel H.: (2002) Matrix engineering for osteogenic differentiation of rabbit periosteal cells using alpha-tricalcium phosphate particles in a three-dimensional fibrin culture. J.Biomed.Mater.Res. 59:690-696

[20] Stern S., Lindenhayn K., Schultz 0., Perka C.: (2000) Cultivation of porcine cells from the nucleus pulposus in a fibrin/hyaluronic acid matrix. Acta Orthop.Scand. 71:496502

[21] Vacanti C. A., Vacanti J. P.: (1994) Bone and cartilage reconstruction with tissue engineering approaches. Otolaryngol.Clin.North Am. 27:263-276

[22] Zekorn T. D., Horcher A., Siebers U., Federlin K., Bretzel R. G.: (1999) Synergistic effect of microencapsulation and immunoalteration on islet allograft survival in bioartificial pancreas. J.Mol.Med. 77:193-198 